\title{
Gene Expression Study in Positron Emission Tomography-Positive Abdominal Aortic Aneurysms Identifies CCL18 as a Potential Biomarker for Rupture Risk
}

\author{
Audrey Courtois, ${ }^{1,2,3}$ Betty V Nusgens, ${ }^{2}$ Roland Hustinx ${ }^{4}$ Gauthier Namur, ${ }^{4,5}$ Pierre Gomez, ${ }^{5}$ \\ Helena Kuivaniemi, ${ }^{6,7}$ Jean-Olivier Defraigne, ${ }^{3}$ Alain C Colige, ${ }^{2^{*}}$ and Natzi Sakalihasan ${ }^{1,4^{*}}$
}

${ }^{1}$ Surgical Research Center, GIGA-Cardiovascular Science Unit, University of Liège, Liège, Belgium; ${ }^{2}$ Laboratory of Connective Tissues Biology, GIGA-Research (GIGA-R), University of Liège, Liège, Belgium; ${ }^{3}$ Department of Cardiovascular and Thoracic Surgery, University Hospital of Liège (CHU), University of Liège, Liège, Belgium; ${ }^{4}$ Department of Nuclear Medicine, University Hospital of Liège (CHU), University of Liège, Liège, Belgium; ${ }^{5}$ Department of Nuclear Medicine, St Joseph Hospital (CHC), Liège, Belgium; ${ }^{6}$ The Sigfried and Janet Weis Center for Research, Geisinger Health System, Danville, Pennsylvania, United States of America; and ${ }^{7}$ Department of Surgery, Temple University School of Medicine, Philadelphia, Pennsylvania, United States of America

\begin{abstract}
Rupture of abdominal aortic aneurysm (AAA) is a cause of significant mortality and morbidity in aging populations. Uptake of 18-fluorodeoxyglucose (FDG) detected by positron emission tomography (PET) is observed in the wall of $12 \%$ of AAA (A+), with most of them being symptomatic. We previously showed that the metabolically active areas displayed adventitial inflammation, medial degeneration and molecular alterations prefacing wall rupture. The aim of this study was to identify new factors predictive of rupture. Transcriptomic analyses were performed in the media and adventitia layers from three types of samples: AAA without FDG uptake $(A 0)$ and with FDG uptake $(A+)$, both at the positive spot $\left(A++_{\mathrm{Pos}}\right)$ and at a paired distant negative site $\left(A+{ }_{\text {Neg }}\right)$ of the same aneurysm. Follow-up studies included reverse-transcriptase-polymerase chain reaction (RT-PCR), immunohistochemical staining and enzyme-linked immunosorbent assay (ELISA). A large number of genes, including matrix metalloproteinases, collagens and cytokines as well as genes involved in osteochondral development, were differentially expressed in the $A+{ }_{\text {pos }}$ compared

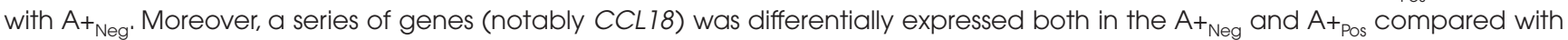
the A0. A significant increase of CCL18 was also found at the protein level in the aortic wall and in peripheral blood of A+ patients compared with A0. In conclusion, new factors, including CCL18, involved in the progression of AAA and, potentially, in their rupture were identified by a genome-wide analysis of PET-positive and -negative human aortic tissue samples. Further work is needed to study their role in AAA destabilization and weakening.
\end{abstract}

Online address: http://www.molmed.org

doi: 10.2119/molmed.2014.00065

\section{INTRODUCTION}

Rupture of abdominal aortic aneurysm (AAA) is a leading cause of death in western societies. Because AAA is generally asymptomatic, the evaluation of rupture risk and its prediction are challenging. Until now, the most commonly used factor to predict AAA rupture is its size (1-3), and a general rule is to perform surgery when the diameter is $>55 \mathrm{~mm}$. Nevertheless, growth of AAA is not linear and highly unpredictable $(2,4)$. In the current practice, a conservative approach is often considered for patients with

*ACC and NS contributed equally to this work.

Address correspondence to Audrey Courtois, Laboratory of Connective Tissue Biology, Avenue de L'Hôpital, B23/+3, 4000 Liège, Belgium. Phone: +32-4-3669293; Fax: +32-4-

3662457; E-mail: a.courtois@ulg.ac.be.

Submitted April 2, 2014; Accepted for publication December 9, 2014; Epub

(www.molmed.org) ahead of print December 10, 2014.

\section{The Feinstein Institute for Medical Research} Empowering Imagination. Pioneering Discovery.

small AAA. However, they may also rupture, whereas many undiscovered aneurysms may grow to a considerable size without rupture. Therefore, diameter is not a fully reliable or specific predictor of the risk of rupture, necessitating the search for new markers.

Uptake of 18-fluorodeoxyglucose (FDG) visualized by positron emission tomography (PET) is currently used to detect hypermetabolic activity of cells seen in tumors and inflammatory processes. Several studies have also shown that FDG-PET can reliably detect leukocyte activities in atherosclerosis $(5,6)$. We previously reported that a focal uptake of FDG is usually observed in the aortic wall of patients with rapidly expanding and symptomatic aneurysms 
that are prone to rupture. Our data also suggest that the FDG-PET-positive spots may correspond to the future site of rupture (7). They are further characterized by the presence of a dense inflammatory infiltrate mainly localized in the adventitia, sometimes organized in tertiary lymphoid organs, and by a marked loss of smooth muscle cells in the media and by an increased expression of molecules involved in the wall remodeling, notably matrix metalloproteinases (MMPs) (7).

This study aims to identify genes involved in this metabolic activation potentially related to rupture. A genomewide transcriptomic analysis was performed on biopsies collected from patients with an FDG uptake both in the positive spot $\left(\mathrm{A}+{ }_{\mathrm{Pos}}\right)$ and in a distant negative site of the same aneurysm $\left(\mathrm{A}+{ }_{\text {Neg }}\right)$. These paired biopsies were further compared with samples collected from AAA patients with no FDG uptake (A0) to discriminate biological alterations associated with FDG uptake, to detect new systemic biomarkers correlated with a higher risk of rupture and to identify new pathways involved in the progression and rupture of AAA.

\section{MATERIALS AND METHODS}

\section{Patients and PET Computed Tomography Image Acquisition}

The investigation conformed to the principals outlined in the Declaration of Helsinki. All patients gave written informed consent for the use of the aortic tissue and serum samples for research. The collection of the human samples was approved by the Ethics Committee of the University Hospital of Liège.

PET computed tomography (PET-CT) data were acquired in two hospitals (CHU and $\mathrm{CHC}$ ), using either a Gemini BB (16slice CT; Philips, Bruxelles, Belgium) or a Discovery LS (16-slice CT; GE Healthcare, Little Chalfont, UK). The image acquisition protocol was performed as previously described (7). The standardized uptake value (SUV) of the positive spot was expressed as the ratio between the uptake in the AAA and the liver (rSUV), with

Table 1. Clinical features of patients without $(A 0)$ and with FDG uptake $(A+)$ used for transcriptomic study.

\begin{tabular}{|c|c|c|c|c|}
\hline Patient ID & AAA size $(\mathrm{mm})$ & rSUV & $\begin{array}{c}\text { RNA pool } \\
\text { (media layer) }\end{array}$ & $\begin{array}{c}\text { RNA pool } \\
\text { (adventitia layer) }\end{array}$ \\
\hline \multicolumn{5}{|l|}{$\mathrm{AO}$} \\
\hline 1 & 60 & 0.33 & $x$ & $x$ \\
\hline 2 & 55 & 0.41 & & $x$ \\
\hline 3 & 57 & 0.18 & $x$ & \\
\hline 4 & 80 & 0.76 & & $x$ \\
\hline 5 & 56 & 0.43 & & $x$ \\
\hline 6 & 53 & 0.58 & $x$ & $x$ \\
\hline Median (IQR) & $56.5(55.3-59.3)$ & $0.40(0.35-0.54)$ & & \\
\hline \multicolumn{5}{|l|}{ A+ } \\
\hline 7 & 52 & 1.04 & $x$ & $x$ \\
\hline 8 & 54 & 0.89 & & $x$ \\
\hline 9 & 40 & 1.93 & $x$ & $x$ \\
\hline 10 & 55 & 1.22 & $x$ & $x$ \\
\hline 11 & 67 & 0.89 & $x$ & \\
\hline 12 & 57 & 0.97 & $x$ & $x$ \\
\hline Median (IQR) & $54.5(52.5-56.5)$ & $1.00^{a}(0.91-1.18)$ & & \\
\hline
\end{tabular}

Standardized uptake value ratio (rSUV): aortic SUVmax/hepatic SUVmax.

${ }^{a} p<0.001$ using Mann-Whitney U test.

each patient acting as his or her own control. Patients with known connective tissue disorders or thoraco-AAAs were excluded from the study.

\section{Tissue and Blood Collection}

The number of samples available for the various analytical procedures was variable depending on clinical or practical reasons (no operation, biopsy not available, sample of poor quality or of too small size to allow all types of analyses).

In A0 patients, a biopsy was collected at the time of surgery in the anterior aneurysmal wall. For A+ patients, two fragments were collected: one at the site of FDG uptake $\left(\mathrm{A}+{ }_{\mathrm{Pos}}\right)$, as localized using anatomical landmarks and CT images as detailed previously (7), and a second taken at a distant site negative for FDG uptake $\left(\mathrm{A}+{ }_{\mathrm{Neg}}\right)$. A full thickness slice of the samples was fixed in $4 \%$ paraformaldehyde for histological and immunohistochemical analysis. When available, the remaining tissue was dissected to separate the media and adventitia, snap-frozen in liquid nitrogen and kept at $-80^{\circ} \mathrm{C}$ until use.

Blood samples were collected from all patients undergoing a PET-CT exam ( $\mathrm{n}=$ 33) and from 12 healthy individuals.
Serum was separated by centrifugation for $10 \mathrm{~min}$ at $3,000 \mathrm{~g}$.

\section{Microarray Analyses}

Among the 12 AAA patients enrolled in the transcriptomic study (Table 1 and Supplementary Table S1), 6 patients had no visible FDG uptake (A0), whereas 6 others presented positive spots $\left(\mathrm{A}+{ }_{\mathrm{Pos}}\right)$ localized within a larger negative area $\left(\mathrm{A}+{ }_{\mathrm{Neg}}\right)$. Demographic information, AAA size and rSUV on these patients are presented in Supplementary Table S1. Tissue samples were ground at liquid nitrogen temperature (Dismembrator apparatus; B. Braun, Berlin, Germany). Total RNA was extracted with TriPure extraction reagen (Roche Diagnostics Corporation, Indianapolis, IN USA) or the RiboPure ${ }^{\mathrm{TM}}$ kit (Ambion [Thermo Fisher Scientific Inc., Waltham, MA, USA]) according to the manufacturer's instructions. Total RNA was quantified (NanoDrop; Isogen Life Science, De Meern, the Netherlands), and its quality (expressed by the RNA quality indicator) was assessed by the Experion Automated Electrophoresis System (Bio-Rad). Three to five RNA samples from each group (adventitia and media of $\mathrm{A} 0, \mathrm{~A}+{ }_{\mathrm{Pos}}$ or $\mathrm{A}+_{\mathrm{Neg}}$ samples) 
with RNA quality indicator values $\geq 7$ were pooled in equal concentration (Table 1). A total of $500 \mathrm{ng}$ pooled RNA was labeled by using the GeneChip Expression 3'-Amplification One-Cycle Target Labeling Kit (Affymetrix, Santa Clara, CA, USA) following the manufacturer's protocol. The cRNA was hybridized to the whole human genome U133Plus2.0 chip (Affymetrix) according to the manufacturer's protocol. The data were generated with the MAS 5.0 algorithm included in the GeneChip Operating Software. Validations of microarray data were performed by real-time quantitative reverse-transcriptase-polymerase chain reaction (qRT-PCR) on individual RNA samples using gene-specific primer pairs (Supplementary Table S2).

The negative $\left(\mathrm{A}+{ }_{\mathrm{Neg}}\right)$ and positive $\left(\mathrm{A}+{ }_{\mathrm{Pos}}\right)$ sites of $\mathrm{A}+$ patients were compared with each other and with samples of the A0 patients. Each comparison was performed separately for the media and the adventitia. A fold-change of $\geq 2$ was used to select differentially expressed genes across the six comparisons. Lists of differentially expressed genes were imported into the DAVID software for analysis $(8,9)$ and examined for biologically relevant associations by using the Gene Ontology (GO) and Kyoto Encyclopedia of Genes and Genome (KEGG) pathway analysis (10). Enriched KEGG pathways were considered to be significant when the modified Fisher exact $p$ value (Ease Score) was $<0.05$. National Center for Biotechnology Information (NCBI) official gene symbols available at http://www.ncbi.nlm.nih.gov were used for annotations.

\section{Real-Time qRT-PCR}

Microarray data were validated for selected genes of interest by real-time qRT-PCR on RNA from individual samples. Briefly, $500 \mathrm{ng}$ total RNA was reverse-transcribed using SuperScript II Reverse Transcriptase (Invitrogen [Thermo Fisher Scientific]). Real-time PCR was performed in a final volume of $20 \mu \mathrm{L}$ containing cDNA corresponding to $1 \mathrm{ng}$ mRNA, $2 \mu \mathrm{mol} / \mathrm{L}$ of each primer and $10 \mu \mathrm{L}$ of the qPCR MasterMix Plus for SYBR-Green (Eurogentec, Liège, Belgium) in the ABI Prism 7300 Sequence Detection System (Applied Biosystems [Thermo Fisher Scientific]). A dilution curve of a reference cDNA was performed for each gene product to calculate the reaction efficiency. Data were further standardized by using the geometrical mean of GAPDH and $\beta 2$ microglobulin expression. These two genes were similarly expressed in all samples (data not shown). Primer sequences are listed in Supplementary Table S2.

\section{Immunological Assays}

For immunohistochemical analyses, 5- $\mu \mathrm{m}$ sections from 22 paraffin-embedded AAA specimens (10 A0, $5 \mathrm{~A}+{ }_{\mathrm{Neg}}$ and $\left.7 \mathrm{~A}++_{\mathrm{Pos}}\right)$ were stained by using antibodies against CCL18 (Abcam ab104867; 1/500; Abcam, Cambridge, UK) and collagen type X (Abcam ab49945; 1/1,000). Briefly, antigen retrieval was achieved with $2.5 \%$ trypsin solution (Gibco, Invitrogen [Thermo Fisher Scientific]) (10 min at $37^{\circ} \mathrm{C}$ ), and the samples were incubated with the primary antibody overnight at $4^{\circ} \mathrm{C}$ (in $10 \%$ bovine serum albumin). Peroxidase-conjugated secondary antibody (Dako, Glostrup, Denmark) was detected using AEC substrate (Dako). The CCL18 labeling was quantified by counting the number of positive cells in 10 fields at high magnification $(\times 400)$. The collagen $\times$ staining was evaluated by computer-assisted image analysis (ImageJ software; National Institutes of Health, Bethesda, MD; [11]). The results were expressed in arbitrary units per surface unit.

For enzyme-linked immunosorbent assay (ELISA), proteins were extracted from separated media and adventitia layers of the aortic wall samples ( $n=10$ for $\mathrm{A} 0$ and $\mathrm{n}=6$ for $\mathrm{A}+$ patients at the $\mathrm{A}+{ }_{\mathrm{Pos}}$ and $\mathrm{A}+{ }_{\mathrm{Neg}}$ sites). Tissues were homogenized in a buffer containing $0.05 \mathrm{~mol} / \mathrm{L}$ Tris- $\mathrm{HCl}$ (pH 7.2), $0.15 \mathrm{~mol} / \mathrm{L} \mathrm{NaCl}$, $0.002 \mathrm{~mol} / \mathrm{L} \mathrm{CaCl}_{2}$ and $2 \mathrm{~mol} / \mathrm{L}$ urea. Serum samples were collected from 12 healthy donors and 33 AAA patients (17 $\mathrm{A} 0$ and $16 \mathrm{~A}+$ ). CCL18 protein levels were measured in tissue extracts and serum by ELISA according to the manufacturer's instructions (R\&D Systems, Minneapolis, MN, USA).

\section{Statistical Analysis}

Results were expressed as the median and the interquartile range (IQR) and considered to be significant at the $5 \%$ critical level $(p<0.05)$ by using the Mann-Whitney $U$ test for unpaired samples or the Wilcoxon signed-rank test for paired samples, as implemented in the Graphpad Prism software (GraphPad Software; GraphPad, La Jolla, CA, USA). Correlation between qRT-PCR and microarray data was calculated by using the Spearman test for nonparametric values as implemented in the GraphPad Prism software.

All supplementary materials are available online at www.molmed.org.

\section{RESULTS}

\section{Transcriptomic Analyses to Compare Expression in FDG-Positive and -Negative Aortic Tissue Samples Taken from the Media and Adventitia Layers}

For the transcriptomic study, we used pooled RNA samples from patients with similar clinical features (Table 1 and Supplementary Table S1). In this population, six patients did not show any significant FDG uptake in the aneurysmal wall (A0 samples), whereas six others presented focal uptake spots $\left(\mathrm{A}+{ }_{\mathrm{Pos}}\right.$ samples) within a much larger region with no uptake $\left(\mathrm{A}+{ }_{\text {Neg }}\right.$ samples). The median aortic diameter was similar in both groups (Table 1). These three types of samples were compared with each other $\left(\mathrm{A}+{ }_{\mathrm{Pos}} / \mathrm{A} 0 ; \mathrm{A}+{ }_{\mathrm{Pos}} / \mathrm{A}+{ }_{\mathrm{Neg}} ; \mathrm{A}+{ }_{\mathrm{Neg}} / \mathrm{A} 0\right)$ in the media and adventitia layers. In this experimental setting, genes that are modulated in a similar way both in the $\mathrm{A}+{ }_{\text {Pos }}$ and $\mathrm{A}+{ }_{\text {Neg }}$ compared with A0 likely reflect general alterations of the wall biology in patients with FDG uptake, whereas comparisons between the $\mathrm{A}+{ }_{\text {Pos }}$ and the $\mathrm{A}+{ }_{\mathrm{Neg}}$ in the same aneurysm give 

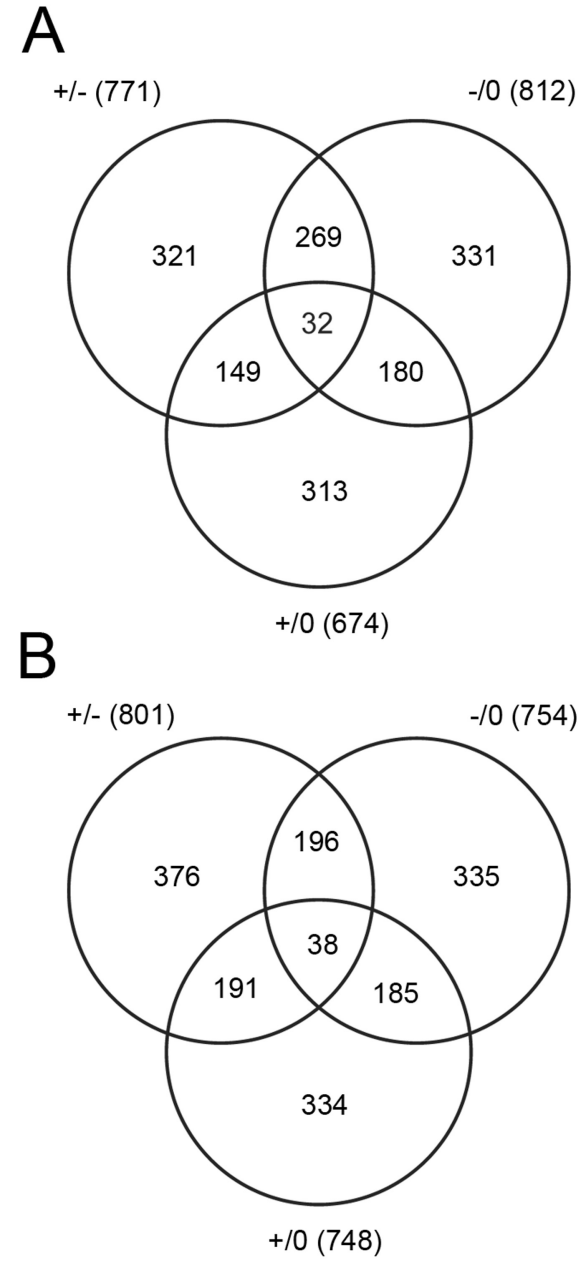

Figure 1. Distribution of differentially expressed genes in the media $(A)$ and adventitia (B) layers of AAA patients. Venn diagram to show the number of genes with differential expression with a foldchange $\geq 2$ when comparing the $\mathrm{A}+{ }_{\mathrm{Neg}}$ to the $\mathrm{AO}$ patients $(-/ 0)$, the $\mathrm{A}+_{\text {Pos }}$ to the $\mathrm{AO}$ $(+/ 0)$ and the $A++_{\text {pos }}$ to the $A+{ }_{\text {Neg }}$ of the same patient (+/-), in the media (A) and the adventitia (B).

insights into more local activation processes at sites potentially more prone to rupture. Using a twofold difference in expression as a cutoff, the six different comparisons identified several hundreds of genes (Figure 1 and Supplementary Table S3; detailed lists are provided in Supplementary Tables S4-S9).

On the basis of pathophysiological knowledge and hypotheses, 13 differentially expressed genes were selected for

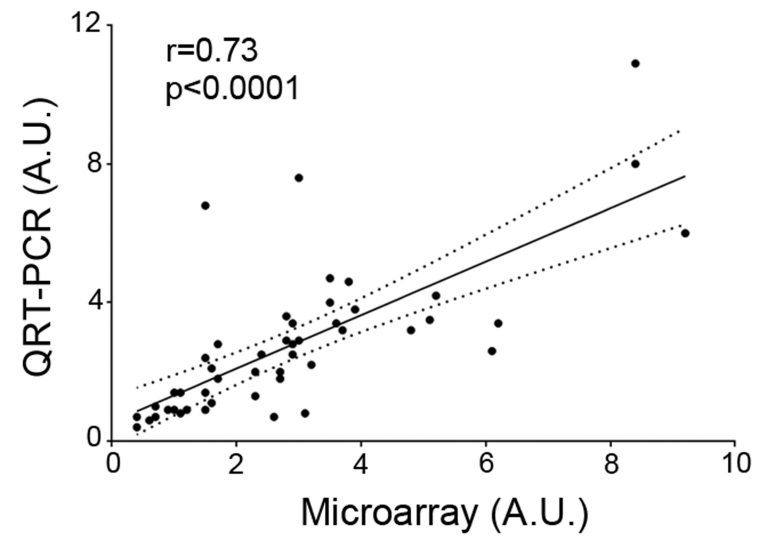

Figure 2. Correlation between the microarray expression data and real-time GRT-PCR data. A linear regression was established between the change in gene expression in the microarray result and the qRT-PCR for 57 comparisons. $r=0.75$ and $p<0.0001$ using the Spearman test. A.U., arbitrary unit.

follow-up studies. Primer pairs (Supplementary Table S2) were designed for validation of microarray data by qRT-PCR by using individual RNA samples of the media and adventitia layers. Among the 58 comparisons reported in Table S10 and S11, the microarray and qRT-PCR results were in agreement in that most of the ratios determined from microarray data were within the range of the values obtained by qRT-PCR. A significant Spearman correlation was found between the microarray and the qRT-PCR results (Figure 2). Altogether, these data demonstrate the reliability of the genome-wide transcriptomic analyses.

\section{Pathway Analysis of Differentially Expressed Genes}

To determine the functions of the differentially expressed genes, we used the DAVID software to classify the genes into biological pathways. The analysis revealed that only approximately $25 \%$ of the differentially expressed genes could be assigned to KEGG pathways (5-15 depending on the comparisons). Many of the enriched KEGG pathways participate to related biological processes, explaining why a significant number of the differentially expressed genes can be found in several pathways.

Comparison between the $\mathrm{A}+{ }_{\text {Pos }}$ and the $\mathrm{A}+{ }_{\mathrm{Neg}}$ showed a significant enrichment of seven pathways in the media and five in the adventitia samples (Tables 2 and 3). The identification of pathways related to the cardiovascular system was expected and further validated our data. The two pathways enriched both in the media and the adventitia (extracellular matrix [ECM] receptor interaction and focal adhesion) are related pathways and probably reflect remodeling of the ECM occurring in the FDG-positive samples. Also of interest was the identification of the cytokine-cytokine receptor interaction pathway potentially indicative of an immune reaction.

Although the $\mathrm{A}+{ }_{\mathrm{Neg}}$ and the A0 samples are both metabolically inactive, their comparison identified 15 enriched KEGG pathways in the media (Supplementary Table S12) and eight in the adventitia (Supplementary Table S13). Again, many pathways are directly related to ECM remodeling, signaling by soluble factors and immune reaction.

\section{Identification of Groups of Genes Relevant to Systemic or Local Alterations of AAA}

Among the differentially expressed genes (Table S4 to S9), those involved in extracellular homeostasis (diverse macromolecules, collagens, proteases) and in inflammatory processes (cytokines) were evaluated more extensively because of 
Table 2. Enriched KEGG pathways in the media of the $\mathrm{A}+{ }_{\mathrm{Pos}}$ versus the $\mathrm{A}+{ }_{\mathrm{Neg}}$ samples.

\begin{tabular}{|c|c|c|c|}
\hline Pathway & $\begin{array}{c}\text { Total } \\
\text { genes } \\
\text { in the } \\
\text { pathway } \\
\text { (n) }\end{array}$ & $\begin{array}{c}\text { Modulated } \\
\text { genes } \\
\text { in } A++_{\text {Pos }} \\
\text { (n) }\end{array}$ & $p$ \\
\hline hsa04512: ECM receptor interaction & 84 & 13 & $1.8 \mathrm{E}-04$ \\
\hline hsa05412: Arrhythmogenic right ventricular cardiomyopathy & 76 & 12 & $3.0 \mathrm{E}-04$ \\
\hline hsa04060: Cytokine-cytokine receptor interaction & 262 & 21 & 7.0E-03 \\
\hline hsa05410: Hypertrophic cardiomyopathy & 85 & 10 & $9.2 \mathrm{E}-03$ \\
\hline hsa04510: Focal adhesion & 201 & 17 & 1.0E-02 \\
\hline hsa04514: Cell adhesion molecules & 132 & 12 & $2.3 \mathrm{E}-02$ \\
\hline hsa05414: Dilated cardiomyopathy & 92 & 9 & 4.0E-02 \\
\hline
\end{tabular}

Significantly enriched KEGG pathways among the differentially expressed genes in the media of $A++_{\text {pos }}$ compared with $A+{ }_{\text {Neg }}$ were identified by using the DAVID software (8). The total number of genes in the pathway, the number of modulated genes observed in our microarray data and the modified Fisher exact $p$ value (Ease Score) are listed. their potential role in the growth and rupture of AAA. Insulinlike growth factors and related molecules were also of interest, since many members of this family were differentially expressed. Selected groups of genes showing systemic or local changes in expression are illustrated for the media (Figure 3) and the adventitia (Figure 4) by using a color coding. Grey boxes represent genes with no change in expression (fold-change $<2$ ), whereas red and green represent increasing levels of upregulation or downregulation, respectively.

Comparisons of the $\mathrm{A}+{ }_{\mathrm{Neg}}$ with A0 (-/0 column in Figures 3 and 4) showed many similarities for transcripts related to ECM turnover and homeostasis (metalloproteases, collagens, osteochondral development) in both the media and adventitia. In contrast, expression of these genes was strongly altered in $\mathrm{A}+{ }_{\mathrm{Pos}}$ compared with the two other conditions (+/ - and $+/ 0$ columns). For example, two collagenases (MMP1 and MMP13) were strongly upregulated in the media and the adventitia of the $\mathrm{A}+{ }_{\mathrm{Pos}}$ samples, although with a high interindividual variability (Figures 3 and 4 ). The expression of elastin and fibulin 5, two major components of the elastic fibers, was reduced, whereas the expression of metalloelastase (MMP12) was strongly upregulated in the adventitia. Some genes related to basement
Table 3. Enriched KEGG pathways in the adventitia of the $\mathrm{A}+{ }_{\mathrm{Pos}}$ versus the $\mathrm{A}+{ }_{\mathrm{Neg}}$ :

\begin{tabular}{|c|c|c|c|}
\hline Pathway & $\begin{array}{l}\text { Total } \\
\text { genes } \\
\text { in the } \\
\text { pathway } \\
\text { (n) }\end{array}$ & $\begin{array}{c}\text { Modulated } \\
\text { genes } \\
\text { in } A+{ }_{\text {Pos }} \\
\text { (n) }\end{array}$ & $p$ \\
\hline hsa04512: ECM receptor interaction & 84 & 16 & 1.9E-06 \\
\hline hsa04510: Focal adhesion & 201 & 21 & 3.1E-04 \\
\hline hsa00380: Tryptophan metabolism & 40 & 8 & 1.3E-03 \\
\hline hsa04270: Vascular smooth muscle contraction & 112 & 12 & 7.8E-03 \\
\hline hsa00350: Tyrosine metabolism & 44 & 6 & $3.8 \mathrm{E}-02$ \\
\hline
\end{tabular}

Significantly enriched KEGG pathways among the differentially expressed genes in the adventitia of $\mathrm{A}+_{\text {Pos }}$ compared with $\mathrm{A}+_{\text {Neg }}$ were identified by using the DAVID software (8). The total number of genes in the pathway, the number of modulated genes observed in our microarray data and the modified Fisher exact $p$ value (Ease Score) are indicated. membrane (LAMA1, LAMA2, LAMB3, NID1) were also differentially regulated in the $\mathrm{A}+{ }_{\mathrm{Pos}}$ sites. The most striking differences were found in the group of collagens, mainly fibril-forming collagens I, III, V and XII. Genes associated with osteochondral development were also aberrantly regulated in the adventitia, including cartilage-specific collagens $\mathrm{X}$ and XI, periostin (POST; a matricellular protein regulating collagen fibril formation and biomechanical properties of connective tissues), transcription factors (RUNX2, $F O X C 1)$ and factors participating in the Wnt signaling (WNT2, WNT5A, FRZB). A different situation was observed for cytokine-related genes, with more similarities between $\mathrm{A}+{ }_{\text {Pos }}$ and $\mathrm{A}+{ }_{\text {Neg }}$ (more gray boxes in the $+/-$ columns and more colored boxes in the $+/ 0$ and $-/ 0$ columns). Of special interest was the high overexpression of IL6 and CCL18 in both the media and adventitia in the entire wall of A+ compared with A0 patients, since these factors are involved in inflammatory processes and could be used as circulating markers.

\section{Increased Protein Production of CCL18 and Collagen Type $X$ in the FDG-Positive Aortic Wall Compared with the FDG-Negative Samples}

Protein expression and tissue localization of type $\mathrm{X}$ collagen were determined by immunostaining on tissue samples, to verify the activation of the osteochondral development pathway in the $\mathrm{A}+{ }_{\mathrm{Pos}}$ adventitia samples (Figure 5A). Type X collagen was localized mainly in the adventitia, and there was significantly more staining in the $\mathrm{A}+{ }_{\text {Pos }}$ tissues compared with the A0 samples (Figure 5C). However, differences were less pronounced than expected from transcriptomic analyses and did not reach statistical significance between $\mathrm{A}+{ }_{\mathrm{Pos}}$ and $\mathrm{A}+{ }_{\mathrm{Neg}}$.

Immunostaining also showed CCL18positive cells mainly in the adventitia (Figure 5B) and in the inner part of the media. A semiquantitative analysis confirmed that there was a significantly increased number of CCL18-positive cells in the $\mathrm{A}+{ }_{\mathrm{Neg}}$ and $\mathrm{A}+{ }_{\mathrm{Pos}}$ groups (Fig- 
Metalloproteases

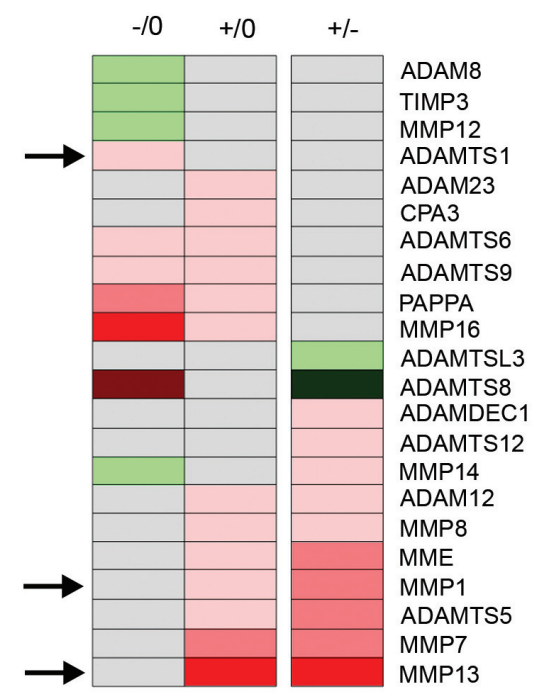

Extracellular matrix

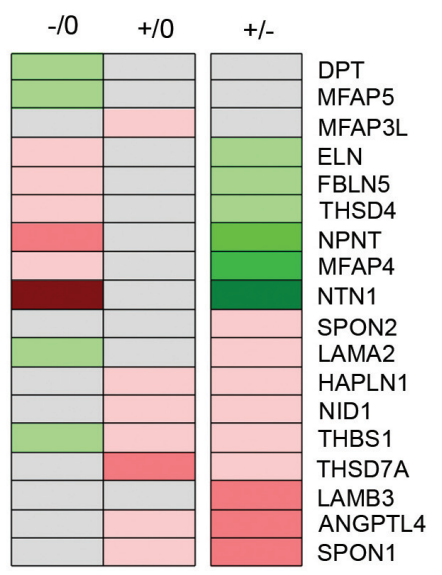

Collagens

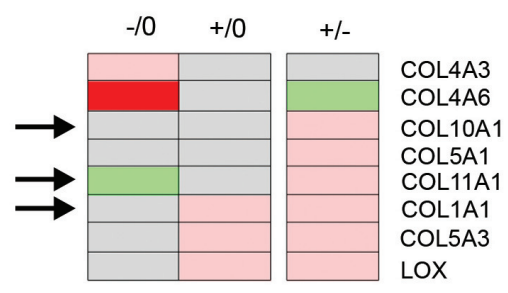

Cytokines
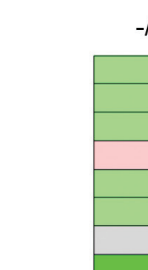

$-10+10+10$

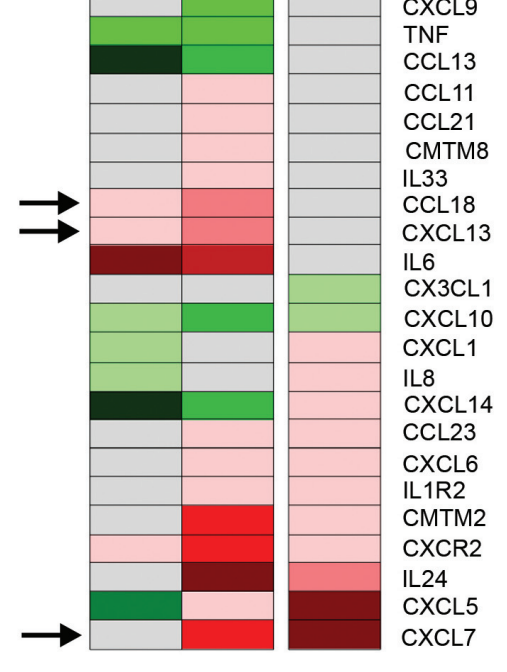

Insulin-like growth factors

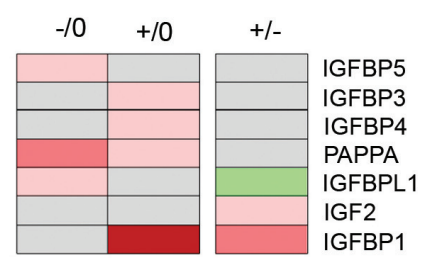

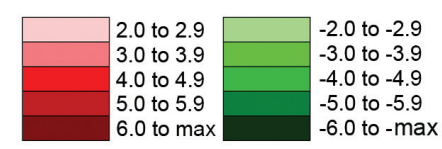

Figure 3. Expression of selected gene families in the aortic media on the basis of microarray analysis. Color codes correspond to the foldchange between the three comparisons: $A+{ }_{N e g}$ compared with $A 0(-/ 0), A+{ }_{\text {Pos }}$ compared with $A 0(+/ 0)$ and $A+{ }_{\text {Pos }}$ Compared with $A+{ }_{N e g}$ $(+/-)$. Arrows designate genes validated by real-time PCR.

ure 5D). These data were validated by ELISA in tissue extracts (Figures 6A, B). The level of CCL18 was not altered in the media of the different groups, whereas it was significantly increased in the $\mathrm{A}+{ }_{\mathrm{Neg}}$ adventitia compared with the A0 patients and was even higher in $\mathrm{A}+{ }_{\text {Pos }}$ than in $\mathrm{A}+{ }_{\mathrm{Neg}}$. The circulating level of CCL18 (Figure 6C) was also measured by ELISA in the serum of 12 healthy donors (clinical characteristics in Supplementary Table S14) and 33 AAA patients (17 A0 and $16 \mathrm{~A}+$ ). CCL18 concentrations were significantly higher in the AAA patients compared with the healthy population. A small but significant difference was also observed between $\mathrm{A} 0$ and $\mathrm{A}+$. The circulating level of CCL18 was not correlated with the diameter of AAA ( $r=0.9$, data not shown).

\section{DISCUSSION}

Pathobiology of AAA is complex and not yet completely understood, although many pathogenic mechanisms have been suggested to participate in AAA progres- sion and rupture (12). Because the majority of AAA patients are asymptomatic, aneurysms are often detected incidentally. However, aneurysms that produce symptoms, such as rapid enlargement and/or severe back or flank pain, are at increased risk of rupture, an often fatal complication. Finding new biological pathways and markers associated with risk for aneurysm rupture by using genome-wide approaches has been the focus of intense research for several years (10,13-15). These findings will 
Metalloproteases

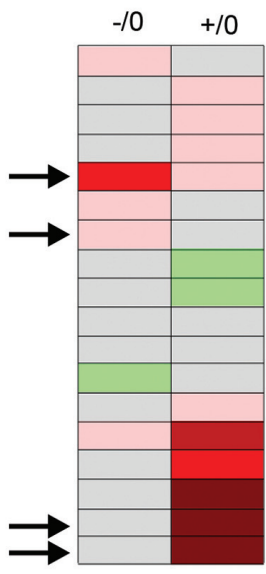

Extracellular matrix

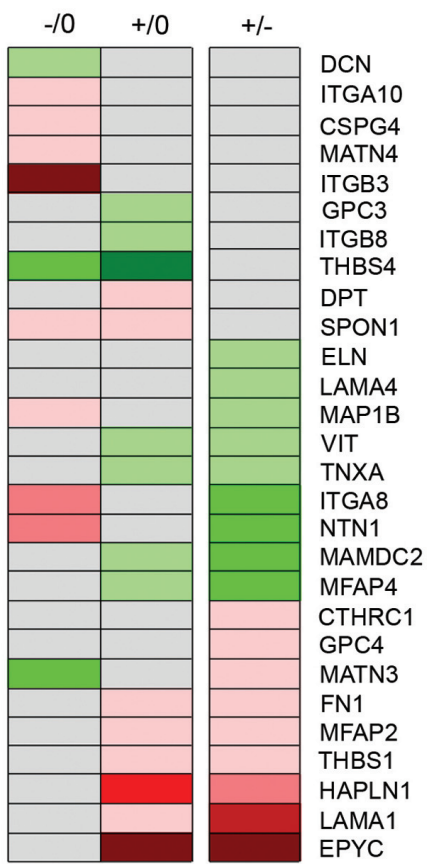

MMP9

MMP11

ADAM12

MMP12

MMP1

MMP13
Collagens
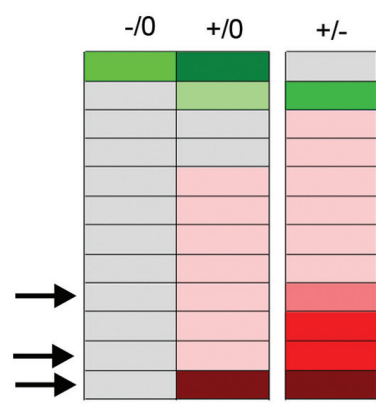

COL28A1

COL4A6

COL15A1

COL8A1

COL3A1

COL5A1

COL5A2

LOX

COL1A1

COL12A1

COL10A1

COL11A1

ADAMDEC 1

\section{Osteochondral development}

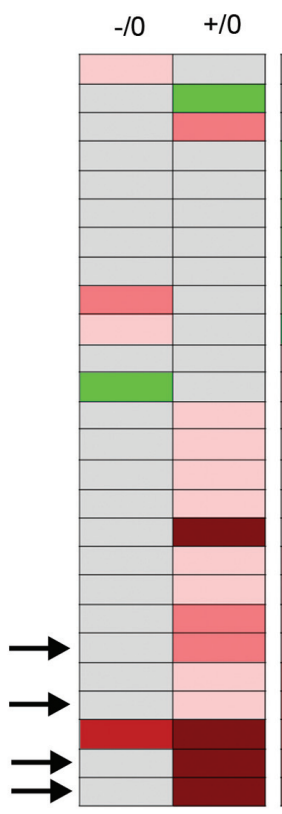

$+/-$

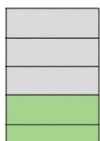

KAZALD1

SOX6

SPP1

CLEC3B

FRZB

OSR2

BMPR1B

WNT5A

FOXC1

IGSF10

ACAN

MATN3

$\mathrm{CDH} 11$

COMP

TNFRSF11B

WNT2

RUNX2

INHBA

CILP2

CRTAC 1

POSTN

COL12A1

COL10A1

IBSP

COL11A1

MMP13
Cytokines

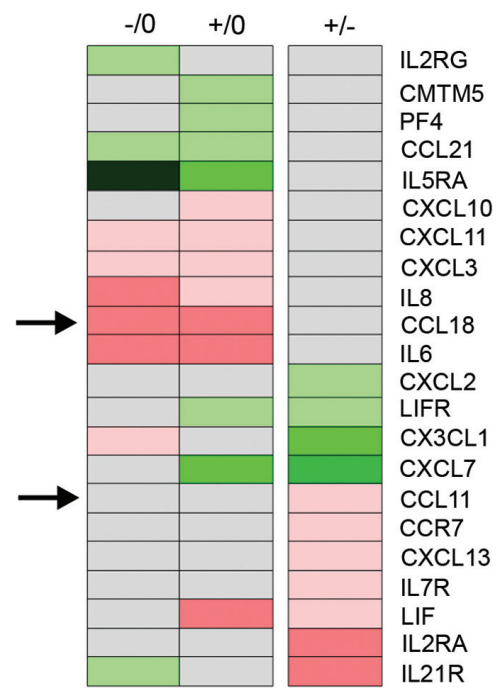

Insulin-like growth factors
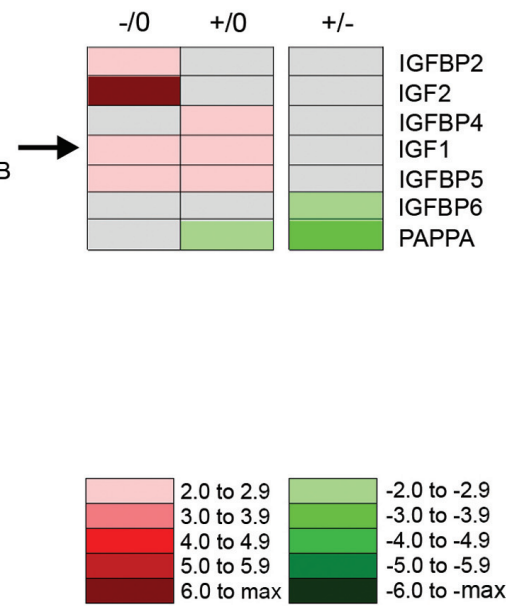

Figure 4. Expression of selected gene families in the aortic adventitia on the basis of microarray analysis. Color codes correspond to the fold-change between the three comparisons: $A+{ }_{\text {Neg }}$ compared with $A 0(-/ 0), A+{ }_{\text {Pos }}$ compared with $A 0(+/ 0)$ and $A++_{\text {Pos }}$ compared with $\mathrm{A}+{ }_{\mathrm{Neg}}(+/-)$. Arrows designate genes validated by real-time PCR.

allow better understanding of rupture risk and predicting this ultimate event. FDG, a metabolic marker used in PET imaging, accumulates preferentially in cells with high metabolic activity, such as tumor cells, activated macrophages and lymphocytes $(16,17)$. Our cohort of patients can be divided into patients with or without significant FDG uptake in the aneurysmal wall. It is worth noting that most of the A0 patients were asymptomatic, whereas a significant number of A+ patients presented warning signs of impending rupture. The positive FDG uptake sites have been shown by us and others to correspond to the maximal peak wall stress (18) and to the site of rupture (19). The novelty of this study was to analyze media and adventitia layers separately from three types of surgical specimen collected either from aneurysms without any significant FDG uptake or from positive aneurysms, at both positive and negative uptake sites. 

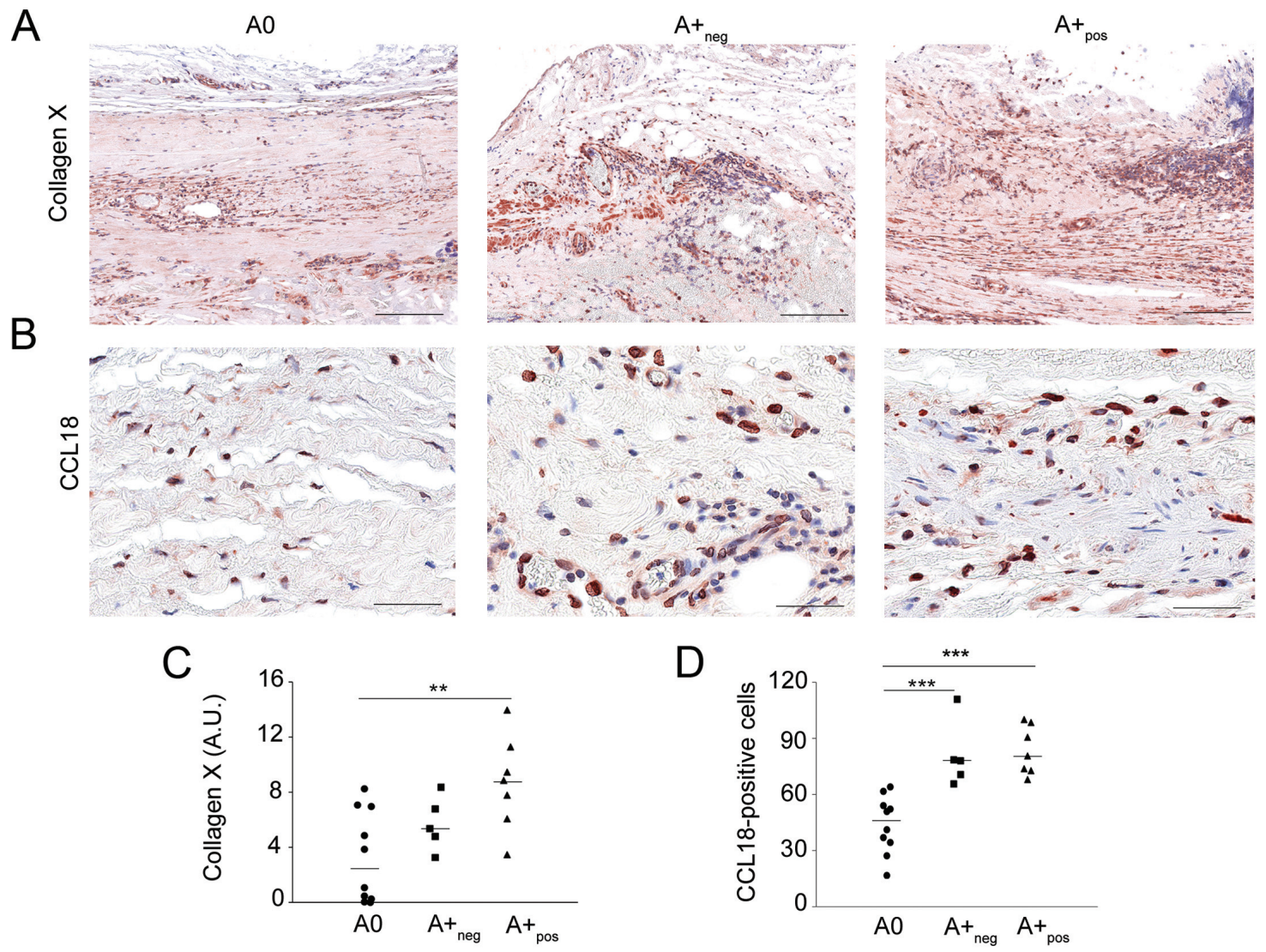

Figure 5. Immunostaining of collagen type $X(A$ and $C$ ) and CCL18 (B and D). Full-thickness aortic wall sections from representative patients with a PET-negative $(A 0)$ or a PET-positive $(A+)$ AAA, at the negative $\left(A+{ }_{N e g}\right)$ and the positive site $\left(A+t_{\text {Pos }}\right)$ are shown. Semiquantitative analysis in $A O(n=10), A++_{N e g}(n=5)$ and $A+_{\text {Pos }}(n=7)$ for the collagen type $X(C)$ staining and CCL18 (D) was carried out. ${ }^{* *} P<0.01$ and ${ }^{* *} P<0.001$ using Mann-Whitney $U$ test or Wilcoxon signed rank test for paired samples. Scale bar in collagen type $X$ sections $=200$ $\mu \mathrm{m}$ and in CCL18 sections $=50 \mu \mathrm{m}$.

Enriched KEGG pathways were identified in the different types of samples. In the media, the cytokine-cytokine receptor interaction pathway was significantly enriched, suggesting the involvement of inflammatory mediators in AAA progression. It is worth noting that some cytokines, such as IL6 and CCL18, were upregulated not only in the $\mathrm{A}+{ }_{\mathrm{Pos}}$ but also in the $\mathrm{A}+{ }_{\mathrm{Neg}}$ compared with the A0. This observation suggests that environmental events and/or systemic factor(s) might affect the whole aneurysmal wall and activate pathways leading to wall degradation and weakening. In agreement with this hypothesis, the majority of A+ patients presented a significantly increased preoperative circulating C-reactive protein (CRP) levels in comparison to A0 pa- tients (7). CCL18 was highly overexpressed throughout the surgical samples of $\mathrm{A}+$ patients $\left(\mathrm{A}+{ }_{\mathrm{Pos}}\right.$ and $\mathrm{A}+{ }_{\mathrm{Neg}}$ sites in the media and the adventitia). Its overexpression was validated by real-time qRTPCR in all the patients of our series and confirmed at the protein level by ELISA. Immunohistochemical analyses of the aortic wall by an anatomopathologist showed that the main CCL18-producing cells are macrophages, with a few endothelial cells and fibroblasts. CCL18 is chemotactic for T and B cells (20), is detected in atherosclerosis plaques in regions rich in macrophages (21) and was also reported as a fibro-inflammatory mediator able to stimulate collagen production by lung and dermal fibroblasts (22). In this study, the plasma levels of
CCL18 were significantly higher in the AAA population compared with healthy individuals, with the highest concentrations being measured in $\mathrm{A}+$ patients. These data suggest that CCL18 could be used as a factor for evaluating the presence and the metabolic activity of AAA in combination with potential markers such as MMP9, which was previously shown to be correlated with the growth of AAA in some studies (23) or with $\mathrm{C}$-reactive protein, which is increased in A+ patients (7).

The inflammatory environment, especially exacerbated in the $\mathrm{A}+{ }_{{ }_{\mathrm{Pos}}}$ certainly contributes to the intense ECM remodeling occurring during AAA progression (24) and ultimately leading to its rupture. According to this hypothesis, Dihlmann 


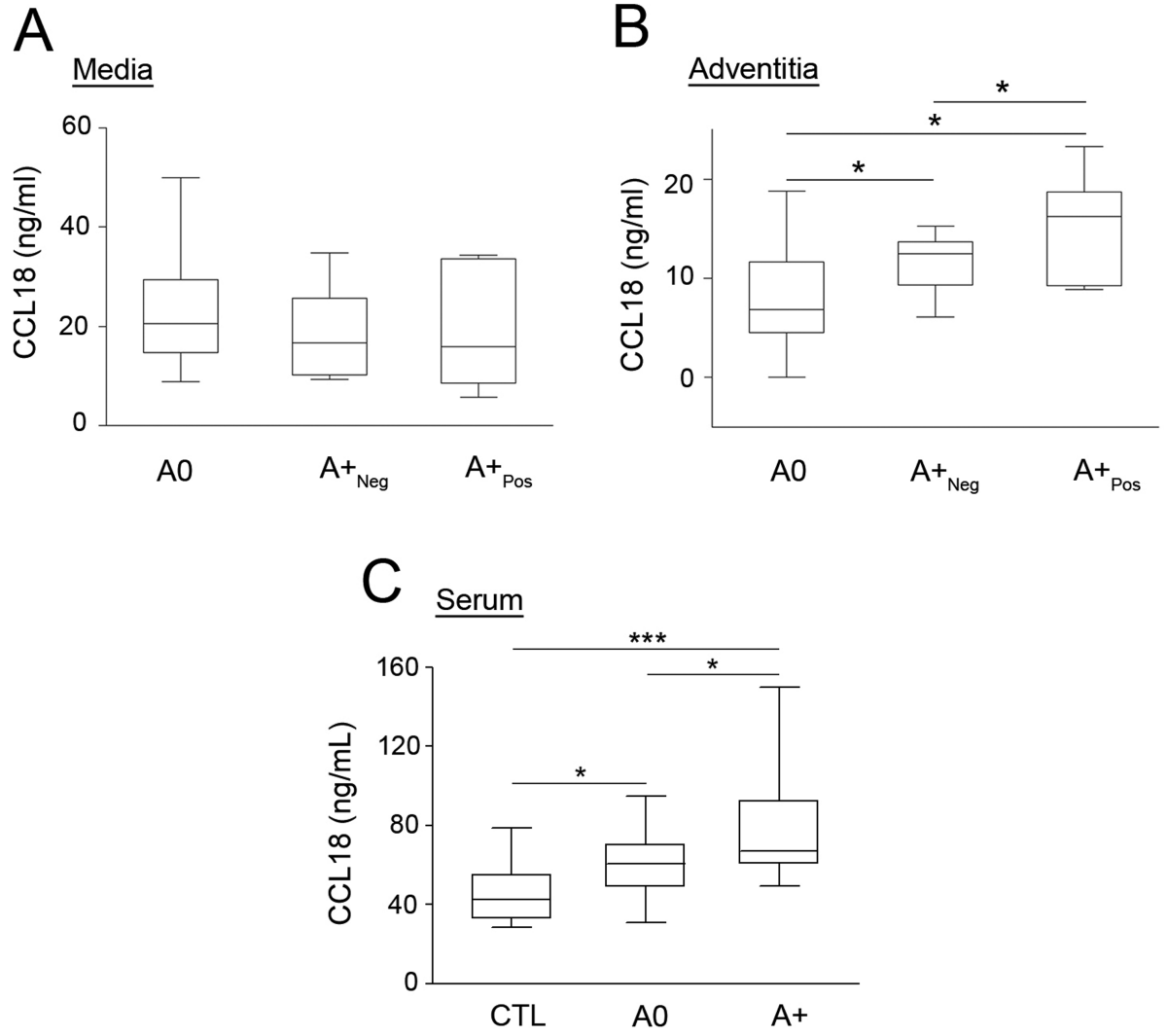

Figure 6. CCL18 protein levels in the aortic wall (A and B) and serum (C) of AAA patients measured by ELISA. Box and whiskers plots of protein levels of CCL18 are shown. CCL18 was measured in the media $(A)$ and adventitia $(B)$ of $A 0(n=11), A+{ }_{N e g}(n=6)$ and $A+{ }_{P o s}$ $(n=7)$ aortic wall samples. Circulating CCL18 (C) was measured in the serum of healthy people $(n=12)$ and AAA patients $(n=33 ; 17$ A0 and $16 A+) \cdot{ }^{*} p<0.05$ and ${ }^{* * *} p<0.001$ using Mann-Whitney $U$ test or Wilcoxon signed rank test for paired samples.

et al. (25) demonstrated recently that the inflammasome process was activated in the AAA wall at the site identified as high rupture risk areas by finite element analysis (25). ECM receptor interaction and focal adhesion KEGG pathways were modified both in the media and in the adventitia of AAA, mostly due to the differential expression of collagens and adhesion molecules such as integrins. On the basis of these data and on previous publications demonstrating the involvement of MMP2 and MMP9 in the growth and rupture of AAA (26-29), the expression of genes involved in ECM accumulation and remodeling were studied in more detail. We found that the expressions of several MMPs, notably collagenases (MMP1, $M M P 8$ and $M M P 13)$, elastases (MMP9 and MMP12), matrilysin (MMP7) and MT1-MMP, the main activator of MMP2, are all upregulated in the $\mathrm{A}+{ }_{\text {Pos }}$ samples. Of special interest, we measured in the adventitia a $>10$-fold increase of MMP1 in the $\mathrm{A}+{ }_{\mathrm{Pos}}$ compared with the $\mathrm{A}+{ }_{\mathrm{Neg}}$, and the expression of $M M P 13$, which was absent in the A0 and weakly expressed in the $\mathrm{A}+{ }_{\mathrm{Neg}}$, was also strongly upregulated in the $\mathrm{A}+{ }_{\mathrm{Pos}}$. MMP13 has been shown to play a crucial role in chronic inflammatory diseases (30) and is increased in AAA (31). The presence of these collagenases at the FDG uptake site could contribute to the degradation of fibrillar collagens I and III, which provide the ultimate tensile strength to the aorta. This scenario could result in a progressive weakening of the wall and finally its rupture (32).
A tentative repair process by the adventitial cells has been previously suggested by the increased expression of COL1A1, a main fibrillar structural component of the adventitia $(33,34)$. We have extended this observation in our microarray analysis. Besides increased expression of type I, III and V fibril-forming collagens, many others collagen types were also overexpressed in the $\mathrm{A}+{ }_{{ }_{\mathrm{Pos}}}$, notably COL10A1 and COL11A1, two collagen types predominantly localized in cartilage and vitreous body. COL10A1 is expressed by hypertrophic chondrocytes during endochondral ossification (35), whereas COL11A1, besides its cartilaginous expression, has been found in peritumoral myofibroblasts (36). Interestingly, a number of other genes involved in cartilage and bone development were also upregulated in the $\mathrm{A}_{{ }_{\mathrm{Pos}}}$ in the adventitia. Among them, periostin (POSTN), a matricellular protein involved in cell adhesion, was first identified in osteoblastic cells (37) and was recently described to be involved in AAA development (38). POSTN localizes mainly in bone but has been also found in fibrous connective tissues subjected to mechanical stress such as heart valves and tendons and in several types of tumors (rev. in 37). It is also overexpressed during vascular injury by proliferative smooth muscle cells to facilitate their migration in the adventitia (39). In a recent proteomic study, POSTN was reported to be increased in AAA (40). Altogether, these original data suggest that cells in the adventitial tissue with a positive FDG uptake possess a specific phenotype leading to the production of an inappropriate ECM. The presence and increased levels of collagen type $X$ protein observed by immunostaining in AAA walls further support this hypothesis. The nature of these cells and their relationship to progenitor lineage need further investigation.

\section{CONCLUSION}

The global gene expression analysis performed here on FDG-positive and -negative aortic tissue samples identified several genes, such as CCL18, po- 
tentially involved in aneurysm progression and rupture. Further studies on larger populations of patients are needed to replicate the findings and assess their clinical value.

\section{ACKNOWLEDGMENTS}

We thank the surgeons and nurses of the Department of Cardiovascular and Thoracic Surgery of the CHU of Liège, the CHC St Joseph and CHR Citadelle for their contribution to the collection of AAA specimens. We thank Benoit Hennuy and the GenoTranscriptomics platform of the GIGA for carrying out the microarray experiments. We thank Joan Somja for analysis of the histological sections. This work was supported by the FP7 European Program Fighting Aneurysmal Diseases number 200647, the Belgian Fonds de la Recherche Scientifique (grant 3.4518.07) and a grant from the University of Liège.

\section{DISCLOSURE}

The authors declare that they have no competing interests as defined by Molecular Medicine, or other interests that might be perceived to influence the results and discussion reported in this paper.

\section{REFERENCES}

1. Sakalihasan N, Limet R, Defawe OD. (2005) Abdominal aortic aneurysm. Lancet. 365:1577-89.

2. Limet R, Sakalihassan N, Albert A. (1991) Determination of the expansion rate and incidence of rupture of abdominal aortic aneurysms. J. Vasc. Surg. 14:540-8.

3. Brown PM, Zelt DT, Sobolev B. (2003) The risk of rupture in untreated aneurysms: the impact of size, gender, and expansion rate. J. Vasc. Surg. 37:280-4.

4. Kurvers H, et al. (2004) Discontinuous, staccato growth of abdominal aortic aneurysms. J. Am. Coll. Surg. 199:709-15.

5. Sakalihasan N, Michel JB. (2009) Functional imaging of atherosclerosis to advance vascular biology. Eur. J. Vasc. Endovasc. Surg. 37:728-34.

6. Rudd JH, Hyafil F, Fayad ZA. (2009) Inflammation imaging in atherosclerosis. Arterioscler. Thromb. Vasc. Biol. 29:1009-16.

7. Courtois A, et al. (2013) 18F-FDG uptake assessed by PET/CT in abdominal aortic aneurysms is associated with cellular and molecular alterations prefacing wall deterioration and rupture. J. Nucl. Med. 54:1740-7.

8. Huang da W, Sherman BT, Lempicki RA. (2009)
Systematic and integrative analysis of large gene lists using DAVID bioinformatics resources. Nat. Protoc. 4:44-57.

9. Huang da W, et al. (2007) DAVID Bioinformatics Resources: expanded annotation database and novel algorithms to better extract biology from large gene lists. Nucleic Acids Res. 35:W169-75.

10. Lenk GM, et al. (2007) Whole genome expression profiling reveals a significant role for immune function in human abdominal aortic aneurysms. BMC Genomics. 8:237.

11. Schneider CA, Rasband WS, Eliceiri KW. (2012) NIH Image to ImageJ: 25 years of image analysis. Nat. Methods. 9:671-5.

12. Michel JB, et al. (2011) Novel aspects of the pathogenesis of aneurysms of the abdominal aorta in humans. Cardiovasc. Res. 90:18-27.

13. Choke E, et al. (2009) Whole genome-expression profiling reveals a role for immune and inflammatory response in abdominal aortic aneurysm rupture. Eur. J. Vasc. Endovasc. Surg. 37:305-10.

14. Hinterseher I, et al. (2013) Novel pathways in the pathobiology of human abdominal aortic aneurysms. Pathobiology. 80:1-10.

15. Tromp G, Kuivaniemi H. (2009) Developments in genomics to improve understanding, diagnosis and management of aneurysms and peripheral artery disease. Eur. J. Vasc. Endovasc. Surg. 38:676-82.

16. Newsholme P, Curi R, Gordon S, Newsholme EA. (1986) Metabolism of glucose, glutamine, long-chain fatty acids and ketone bodies by murine macrophages. Biochem. J. 239:121-5

17. Bos R, et al. (2002) Biologic correlates of (18)fluorodeoxyglucose uptake in human breast cancer measured by positron emission tomography. J. Clin Oncol. 20:379-87.

18. Xu XY, et al. (2010) High levels of 18F-FDG uptake in aortic aneurysm wall are associated with high wall stress. Eur. J. Vasc. Endovasc. Surg. 39:295-301.

19. Sakalihasan N, Hustinx R, Limet R. (2004) Contribution of PET scanning to the evaluation of abdominal aortic aneurysm. Semin. Vasc. Surg. 17:144-53.

20. Schutyser E, Richmond A, Van Damme J. (2005) Involvement of CC chemokine ligand 18 (CCL18) in normal and pathological processes. J. Leukoc. Biol. 78:14-26.

21. Wortmann M, Peters AS, Hakimi M, Bockler D, Dihlmann S. (2014) Glyoxalase I (Glo1) and its metabolites in vascular disease. Biochem. Soc. Trans. 42:528-33.

22. Atamas SP, et al. (2003) Pulmonary and activation-regulated chemokine stimulates collagen production in lung fibroblasts. Am. J. Respir. Cell Mol. Biol. 29:743-9.

23. Golledge J, Tsao PS, Dalman RL, Norman PE. (2008) Circulating markers of abdominal aortic aneurysm presence and progression. Circulation. 118:2382-92.

24. Liapis CD, Paraskevas KI. (2003) The pivotal role of matrix metalloproteinases in the development of human abdominal aortic aneurysms. Vasc. Med. 8:267-71.
25. Dihlmann S, et al. (2014) Increased expression and activation of absent in melanoma 2 inflammasome components in lymphocytic infiltrates of abdominal aortic aneurysms. Mol. Med. 20:230-7.

26. Sakalihasan N, Delvenne P, Nusgens BV, Limet R, Lapiere CM. (1996) Activated forms of MMP2 and MMP9 in abdominal aortic aneurysms. J. Vasc. Surg. 24:127-33.

27. Petersen E, Wagberg F, Angquist KA. (2002) Proteolysis of the abdominal aortic aneurysm wall and the association with rupture. Eur. J. Vasc. Endovasc. Surg. 23:153-7.

28. Wilson WR, et al. (2006) Matrix metalloproteinase- 8 and -9 are increased at the site of $a b-$ dominal aortic aneurysm rupture. Circulation. 113:438-45.

29. Aziz F, Kuivaniemi H. (2007) Role of matrix metalloproteinase inhibitors in preventing abdominal aortic aneurysm. Ann. Vasc. Surg. 21:392-401.

30. Wernicke D, Seyfert C, Gromnica-Ihle E, Stiehl P. (2006) The expression of collagenase 3 (MMP-13) mRNA in the synovial tissue is associated with histopathologic type II synovitis in rheumatoid arthritis. Autoimmunity. 39:307-13.

31. Tromp G, et al. (2004) Elevated expression of matrix metalloproteinase-13 in abdominal aortic aneurysms. Ann. Vasc. Surg. 18:414-20.

32. Loftus IM, Thompson MM. (2002) The role of matrix metalloproteinases in vascular disease. Vasc. Med. 7:117-33.

33. Defawe OD, et al. (2003) TIMP-2 and PAI-1 mRNA levels are lower in aneurysmal as compared to athero-occlusive abdominal aortas. Cardiovasc. Res. 60:205-13.

34. Sakalihasan N, Heyeres A, Nusgens BV, Limet R, Lapiere CM. (1993) Modifications of the extracellular matrix of aneurysmal abdominal aortas as a function of their size. Eur. J. Vasc. Surg. 7:633-7.

35. Gouttenoire J, et al. (2004) Modulation of collagen synthesis in normal and osteoarthritic cartilage. Biorheology. 41:535-42.

36. Garcia-Ocana M, et al. (2012) Characterization of a novel mouse monoclonal antibody, clone 1E8.33, highly specific for human procollagen 11A1, a tumor-associated stromal component. Int. J. Oncol. 40:1447-54.

37. Merle B, Garnero P. (2012) The multiple facets of periostin in bone metabolism. Osteoporos. Int. 23:1199-212.

38. Yamashita O, et al. (2013) Periostin links mechanical strain to inflammation in abdominal aortic aneurysm. PLoS One. 8:e79753.

39. Lindner V, Wang Q, Conley BA, Friesel RE, Vary CP. (2005) Vascular injury induces expression of periostin: implications for vascular cell differentiation and migration. Arterioscler. Thromb. Vasc. Biol. 25:77-83.

40. Didangelos A, et al. (2011) Extracellular matrix composition and remodeling in human abdominal aortic aneurysms: a proteomics approach. Mol. Cell. Proteomics. 10:M111.008128. 